\title{
Epitope Mapping of Type VII Collagen

\author{
Identification of Discrete Peptide Sequences Recognized by Sera \\ from Patients with Acquired Epidermolysis Bullosa
}

\author{
Jean-Christophe Lapiere, David T. Woodley, * M. Gabriela Parente, * Toshiroh Iwasaki, * \\ Kimberly C. Wynn, * Angela M. Christiano, and Jouni Uitto \\ Departments of Dermatology, and Biochemistry and Molecular Biology, Jefferson Medical College, and Section of Molecular \\ Dermatology, Jefferson Institute of Molecular Medicine, Thomas Jefferson University, Philadelphia, Pennsylvania 19107; \\ and ${ }^{*}$ Department of Dermatology, Stanford University School of Medicine, Stanford, California 94305
}

\section{Abstract}

Epidermolysis bullosa acquisita (EBA) is an acquired blistering skin disease characterized by the presence of IgG autoantibodies that recognize type VII (anchoring fibril) collagen. In this study, we have mapped the antigenic epitopes within the type VII collagen $\alpha$ chain by Western immunoblotting analysis with sera from 19 patients with EBA, using bacterial collagenase- or pepsin-resistant portions of type VII collagen and a panel of 12 recombinant fusion proteins corresponding to $\sim 80 \%$ of the primary sequence of the $\alpha 1$ (VII) collagen polypeptide. These studies identified four major immunodominant epitopes localized within the amino-terminal, noncollagenous (NC-1) domain. In addition to EBA, sera from three patients with bullous systemic lupus erythematosus (BSLE) were tested. The pattern of epitopes recognized by these sera were similar to those noted with EBA, suggesting that the same epitopes could serve as autoantigens in both blistering conditions. In contrast, sera from healthy controls or from patients with unrelated blistering skin diseases did not react with type VII collagen epitopes. Collectively, the results indicate that the immunodominant epitopes in EBA and BSLE lie within the noncollagenous regions of type VII collagen. The precise role of the circulating autoantibodies in the pathogenesis of these blistering diseases remains to be elucidated. Conceivably, however, such antibodies could disrupt the assembly of type VII collagen into anchoring fibrils and/or interfere with their interactions with other extracellular matrix molecules within the cutaneous basement membrane zone. (J. Clin. Invest. 1993. 92:18311839.) Key words: antigenic determinants • blistering skin disorders • bullous systemic lupus erythematosus • epidermolysis bullosa acquisita $\bullet$ type VII collagen

\section{Introduction}

Epidermolysis bullosa (EB $)^{1}$ is a group of both acquired and heritable disorders characterized by blistering of the skin and

Address reprint requests to Dr. David T. Woodley, Department of Dermatology, Tarry 4-711, Northwestern University School of Medicine, 303 East Chicago, Chicago, IL 60611.

Received for publication 25 September 1992 and in revised form 28 April 1993.

1. Abbreviations used in this paper: EB, epidermolysis bullosa; EBA, EB acquista; BSLE, bullous systemic lupus erythematosus.

J. Clin. Invest.

(c) The American Society for Clinical Investigation, Inc. 0021-9738/93/10/1831/09 \$2.00

Volume 92, October 1993, 1831-1839 mucous membranes (1-3). EB can be classified into three major categories on the basis of the level of tissue separation: (a) simplex, nonscarring forms; (b) junctional forms; and (c) dystrophic, scarring forms. Among the heritable forms of EB, both autosomal dominant and autosomal recessive inheritance patterns can be recognized. In addition to inherited $\mathrm{EB}$, an acquired autoimmune form of EB, known as EB acquisita (EBA), has been delineated $(4,5)$. Patients with EBA have IgG autoantibodies which recognize type VII collagen (6), the major component of anchoring fibrils located at the dermal-epidermal basement membrane zone $(7,8)$. The clinical appearance of EBA patients and the histology of their cutaneous lesions are very reminiscent of hereditary dystrophic EB (6). Furthermore, ultrastructural studies demonstrated a dramatic paucity of anchoring fibrils within the dermal-epidermal junction in both EBA and heritable forms of dystrophic EB $(1-3,9,10)$.

Type VII collagen is a homotrimer consisting of three identical subunit polypeptides, $\alpha$ l (VII) (8). Recent cloning of human $\alpha 1$ (VII) collagen complementary and genomic DNAs has allowed elucidation of the primary structure of type VII collagen $(8,11,12)$. The amino acid sequence information deduced from nucleotide sequences has predicted that type VII collagen consists of a $\sim 145 \mathrm{kD}$ central collagenous domain characterized by a repeating Gly-X-Y amino acid sequence. However, the Gly-X-Y repeat sequence is interrupted by several imperfections, including a 39-amino acid noncollagenous segment (8). The collagenous domain is flanked by a large, $\sim 145 \mathrm{kD}$ noncollagenous domain, NC-1, and by a smaller, $\sim 20 \mathrm{kD}$ noncollagenous domain, NC-2. Information from overlapping complementary and genomic DNA clones has unequivocally demonstrated that the NC-1 domain is the amino-terminal, while the NC-2 segment resides at the carboxy-terminal end of the molecule (12). In the extracellular space, type VII collagen molecules form antiparallel dimers stabilized by disulfide bonding, and a portion of the NC-2 domain is proteolytically removed. Several of these antiparallel dimer molecules then laterally aggregate to form the anchoring fibrils. Thus, the tissue form of type VII collagen consists of a central collagenous domain, a large amino-terminal NC-1 domain, and a truncated carboxy-terminal NC-2 domain (8).

Initial characterization of the autoantibodies in EBA suggested that they were directed against type VII collagen (13). Evidence for the specificity of EBA autoantibodies to type VII collagen sequences is further supported by recent cloning of the first human type VII collagen cDNA K-131 from a keratinocyte $\lambda g t 11$ expression library by screening with the IgG fraction of the serum from a patient with EBA (11). However, there is no further information regarding the subdomains or epitopes recognized by these autoantibodies. 
In this study, we have developed a panel of fusion proteins corresponding to different segments of human type VII collagen. These fusion proteins have been used for epitope mapping of antibodies in 19 patients with EBA. The results identified several discrete regions within the $\alpha 1$ (VII) collagen chain which contain epitopes recognized by these antibodies.

\section{Methods}

Patients' sera and antibodies. The sera used in these experiments were obtained from 19 patients with EBA. These patients all had an active, chronic, mechanobullous disorder with subepidermal blisters, as assessed by routine light microscopy of lesional skin. IgG deposits were detected within the sub-lamina densa region of the basement membrane zone below the epidermis of their skin using direct immunoelectron microscopy in all patients. Anti-type VII collagen antibodies circulating in their serum were identified by Western immunoblotting against type VII collagen extracted from human skin or purified from WISH cell-conditioned medium $(6,13,14)$. Because sera from patients with bullous systemic lupus erythematosus (BSLE) have been shown to have autoantibodies to type VII collagen (15), three sera from patients with BSLE were tested. Controls included sera from healthy normal individuals of different ages $(n=5)$, patients with bullous pemphigoid $(n=3)$, pemphigus vulgaris $(n=2)$, pemphigus foliaceus $(n=2)$, and one patient with pemphigus paraneoplastica $(16,17)$

In addition to human sera, two mouse monoclonal antibodies, L3D and $\mathrm{H} 3 \mathrm{~A}(14,18)$, recognizing human type VII collagen, were tested to determine their epitope targets within the type VII collagen molecule. The controls for the latter antibodies were monoclonal antibodies to human keratin (a generous gift from Dr. Tung-Tien Sun, Department of Dermatology, New York University School of Medicine).

Western immunoblotting analysis. Type VII collagen (also known as the EBA antigen) extracted from human skin (15) or biosynthetically derived from the conditioned medium of WISH cell cultures (14), as well as pepsinized human type VII collagen (13) (Telios Pharmaceuticals, San Diego, CA), or bacterial collagenase-degraded type VII collagen were subjected to SDS-PAGE and Western immunoblotting as previously described $(6,19,20)$. All sera were tested at dilutions of $1: 100$ or greater. In various experiments, $10 \%$ polyacrylamide gels or 4-20\% gradient polyacrylamide gels were used to examine a wide size range of potentially reactive proteins. In experiments with purified fusion proteins ( see below), $3 \mu \mathrm{g}$ of each protein was electrophoresed and blotted. In some experiments with pepsinized type VII collagen, the gel lanes were overloaded with $10 \mu \mathrm{g}$ of protein in order to insure that the amount of protein was sufficient to conclude definitively that the reaction with the sera was negative.

Epitope selection and immunoabsorption of antibodies. To confirm the identity of each epitope and exclude a repeating motif within the fibronectin type III-like repeat of the NC-1 domain (Fig. 1), immunoadsorption and epitopes selection were performed. Immunoreactive fusion proteins were blotted on nitrocellulose filters and incubated with EBA patients' serum diluted 1:100 (EBA 1 and EBA 7 of Fig. 3 and Table II). EBA sera (containing unbound antibodies) were recouped and used for Western immunoblotting against the panel of fusion proteins. Antibodies bound to the fusion protein immobilized onto the nitrocellulose filters were eluted as described (11) and used for indirect immunofluorescence (6).

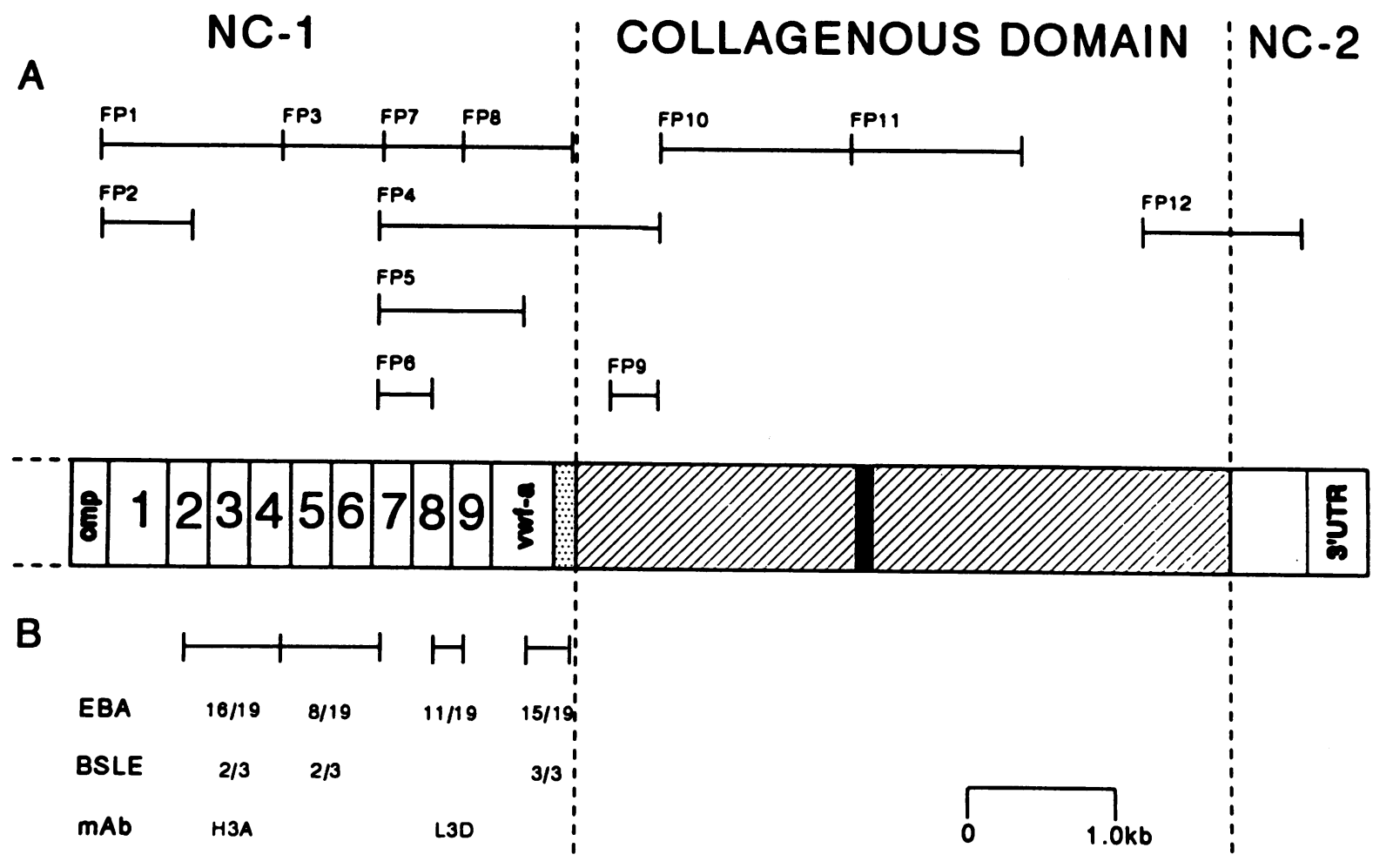

Figure 1. Schematic representation of the positions of recombinant fusion proteins used for epitope mapping with respect to type VII collagen. $(A)$ The positions of 12 fusion proteins which correspond to the noncollagenous domains NC-1 and NC-2, or to the collagenous domain of the protein are indicated. The NC-1 domain consists of several subdomains, including a region with homology to the cartilage matrix protein $(\mathrm{cmp})$, 9 consecutive segments with homology to fibronectin type III domains (1-9), and the A domain of von Willebrand factor ( $v w f-a)$. Cysteineproline-rich region $(\square)$, and the collagenous domain (ש्C), as well as the 39-amino acid noncollagenous interruption in the central portion of the collagenous domain $(\square)$ are indicated. $(B)$ The number of sera positive among the 19 EBA and 3 BSLE sera is indicated by the regions tested using a combination of the recombinant fusion proteins. Also, the areas recognized by two monoclonal antibodies, H3A and L3D are indicated. 
Table I. Characteristics of the Recombinant Fusion Proteins Corresponding to Type VII Collagen Sequences

\begin{tabular}{|c|c|c|c|c|c|c|c|}
\hline & \multirow{2}{*}{$\begin{array}{l}\text { Fusion } \\
\text { protein }\end{array}$} & & & \multicolumn{2}{|c|}{ Amino acid sequence } & \multicolumn{2}{|c|}{$M_{r}(\mathrm{kD})$} \\
\hline & & \multicolumn{2}{|c|}{ Cloning site } & Amino-terminus & Carboxyl-terminus & Thrombin cleaved & Uncleaved \\
\hline \multirow{9}{*}{ NC-1 } & FP1 & BamHI & EcoRI & SILRTLLPLV. . . . & . . . REPPETPLAV & 46 & 72 \\
\hline & FP2 & BamHI & EcoRI & SILRTLLPLV. . . . & . . .GATGYRVTWR & 20 & 46 \\
\hline & FP3 & BamHI & EcoRI & ETPLAVPGLR. . . . & . . . .MRHQILPGNT & 26.5 & 52.5 \\
\hline & FP4 & EcoRI & EcoRI & GGPMRHQILP. . . . & . . . GSPGPQGPVG & 67.5 & 93.5 \\
\hline & FP5 & EcoRI & EcoRI & GGPMRHQILP. . . . & . . . RYMLAPDAPG & 43.5 & 69.5 \\
\hline & FP6 & EcoRI & EcoRI & GGPMRHQILP. . . . & . . .ATQYRVRLSV & 13.5 & 39.5 \\
\hline & FP7 & BamHI & EcoRI & HQILPGNTDS. . . . & . . . EPGVSYIFSL & 23 & 49 \\
\hline & FP8 & EcoRI & EcoRI & VSYIFSLTPV. . . . & . . . .PEPCPVYCPK & 26.5 & 52.5 \\
\hline & FP9 & SmaI & EcoRI & GERGPRGPKG. . . . & . . . GSPGPQGPVG & 10.5 & 36.5 \\
\hline \multirow[t]{2}{*}{ Collagenous domain } & FP10 & EcoRI & EcoRI & KGDSEDGAPA. . . . & . . . EPGSVPNVDR & 46.5 & 72.5 \\
\hline & FP11 & BamHI & EcoRI & SVPNVDRLLE. . . . & . . .GAPGGLAGDL & 40.5 & 66.5 \\
\hline $\mathrm{NC}-2$ & FP12 & BamHI & EcoRI & GSPGKDGVPG. . . . & . . . QSQGTGTAQD & 60.5 & 86.5 \\
\hline
\end{tabular}

The positions of the fusion proteins are indicated in Fig. $1 \mathrm{~A}$. The cloning sites in the pGEX vector, and the amino-terminal and carboxyl-terminal amino acid sequences at the ends of the peptide sequences encoded by the insert cDNA are indicated. The $M_{\mathrm{r}}$ 's of the fusion proteins with and without thrombin clevage, which liberates the peptide segment corresponding to type VII collagen sequences from the GST, are indicated on the right.

Immunofluorescence using epitope-selected antibodies on fusion protein was performed on normal human $1 \mathrm{M} \mathrm{NaCl}$ salt split skin in which dermis is separated from epidermis by fracture of the dermalepidermal junction as previously described (6).

Constructs for expression of recombinant fusion proteins. To generate fusion proteins corresponding to discrete segments within the $\alpha 1$ (VII) collagen polypeptides, a series of constructs which express well-defined fusion proteins were developed (Fig. $1 A$ and Table I). First, a construct containing an insert, FP4 corresponding to the $\alpha 1$ (VII) collagen cDNA K-131 (11), was developed. For this purpose, the insert was isolated from the Bluescript plasmid by EcoRI restriction enzyme digestion. The insert was then subcloned into a pGEX vector (Pharmacia, Inc., Piscataway, NJ; modified by Dr. George Giudice, Medical College of Wisconsin) containing a Taq promoter and the glutathione-S-transferase (GST) gene, followed downstream by a thrombin cleavage site, a multiple cloning site for SmaI, BamHI, and EcoRI restriction enzymes, and a region with translation termination codons in all three reading frames $(21)$. This modified pGEX construct contained the EcoRI site in the correct reading frame to insure proper translation of the insert cDNA into protein.

Secondly, digestion of the cDNA FP4 with either NarI, StyI, or Smal restriction endonuclease generated constructs FP5, FP6, or FP9, respectively. The digestion of FP4 with NarI was performed in the Bluescript vector, and with StyI in the modified pGEX vector. For digestion with SmaI, the cDNA FP4 was subcloned into the EcoRI site of the original pGEX-2T vector, followed by digestion with SmaI and religation.

Thirdly, a series of double-stranded cDNAs corresponding to different regions of the $\alpha 1$ (VII) collagen polypeptide was developed by PCR amplification of segments in the $\alpha 1$ (VII) collagen cDNAs $(8,11,12)$. The upstream primers were synthesized to contain a BamHI site and the downstream primers contained an EcoRI site in their $5^{\prime}$ ends, which allowed cloning of the CDNAs into the modified pGEX vector in correct orientation and reading frame, generating constructs FP1, 3, 7, $8,10,11$, and 12 (Table I). In case the cDNA contained an internal BamHI site, both primers contained EcoRI sites in their 5 ' ends. One of the PCR-generated constructs, FP1, was digested with the PpuMI restriction enzyme to yield the construct FP2 (Fig. $1 A$ ). The correct orientation and reading frame of each insert cloned into the pGEX vector were determined by nucleotide sequencing of the cloning junctions (22).
The constructs were transfected into Escherichia coli (DH5 $\alpha$ ) and grown to an OD of $\sim 0.5$ at $600 \mathrm{~nm}$ in 1 liter of Luria broth medium at $37^{\circ} \mathrm{C}$. Subsequently, isopropyl $\beta$-D-thiogalactopyranoside (in $0.5 \mathrm{mM}$ final concentration) was added, and the incubations were continued for another $7 \mathrm{~h}$. Bacteria were recovered by low-speed centrifugation and suspended in lysis buffer $\left(150 \mathrm{mM} \mathrm{NaCl}, 16 \mathrm{mM} \mathrm{NaH}_{2} \mathrm{HPO}_{4}, 4\right.$ $\mathrm{mM} \mathrm{Na} \mathrm{HPO}_{4}, 1 \mathrm{mM}$ DTT, $1 \mathrm{mM}$ EDTA, $1 \%$ Triton X100, $1 \%$ Tween 20, 1 mM PMSF, pH 7.2), followed by gentle sonication for 30 $\mathrm{s}$ on ice (21). The sonicate was centrifuged at $10,000 \mathrm{~g}$ for $10 \mathrm{~min}$, and the supernatant was collected. The pellet was reextracted using the same procedure, and the supernatants were combined.

To purify the fusion protein expressed in bacteria, the supernatants were applied to a column consisting of agarose beads linked to glutathione in S-linkage (Sigma Chemical Co., St. Louis, MO). The bound proteins were eluted from the column by the addition of $5 \mathrm{mM}$ reduced glutathione (Sigma) in $50 \mathrm{mM}$ Tris, pH 8.0 (21). The eluted proteins were collected in 1-ml fractions, and the peak fractions of proteins, determined by a commercial kit (Bio-Rad Laboratories, Richmond, CA ) or by absorbance at $280 \mathrm{~nm}$, were saved and used for Western blot analyses.

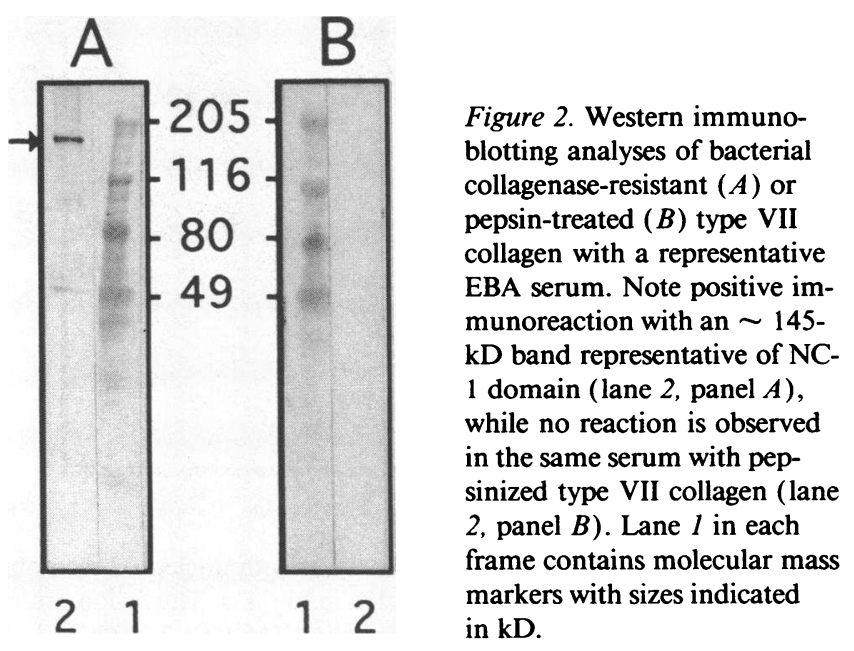




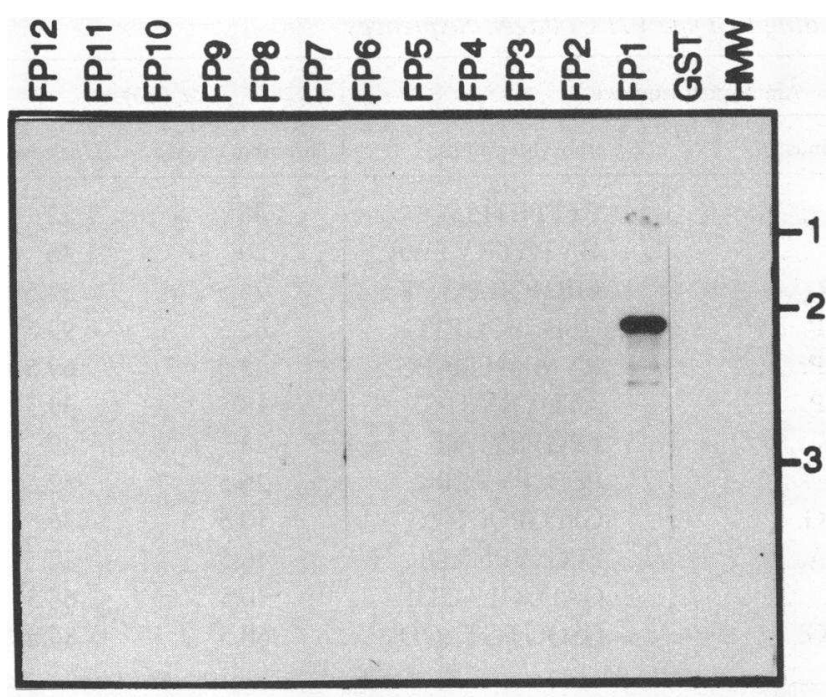

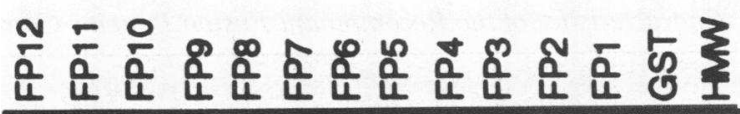

\section{H3A}

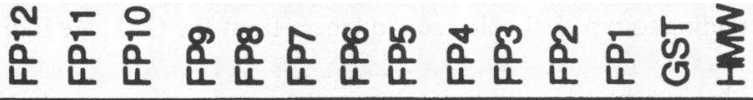

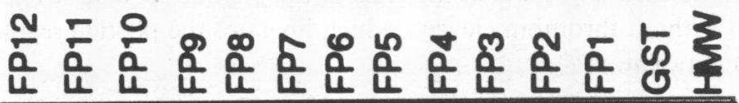

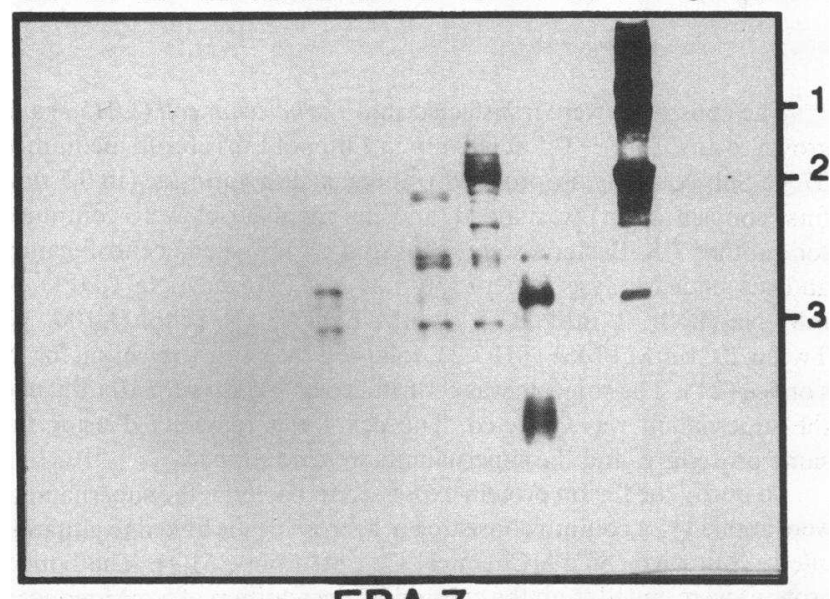

EBA 7

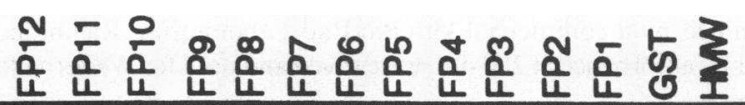

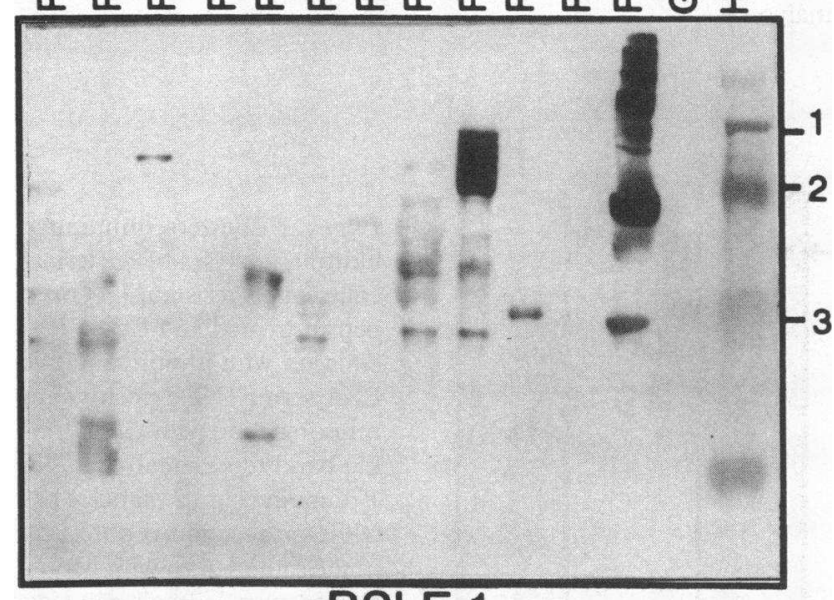

BSLE 1

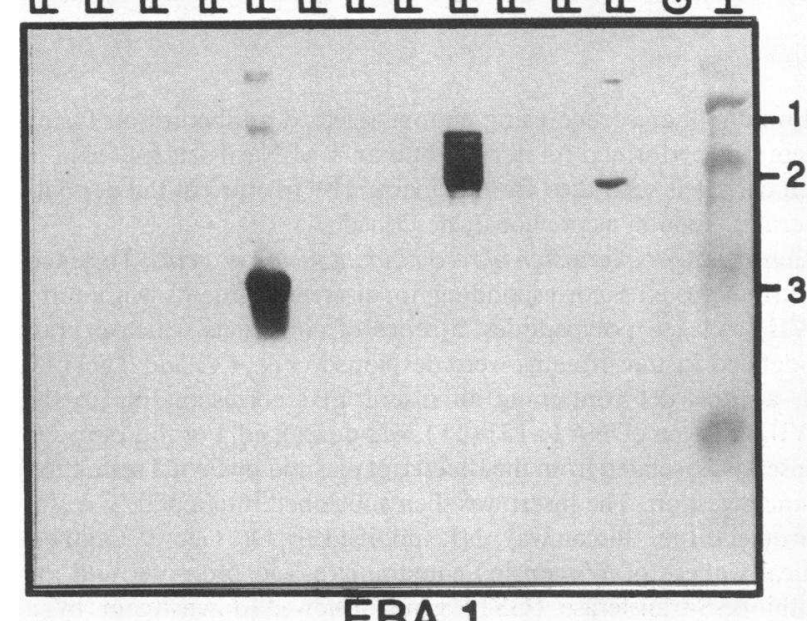

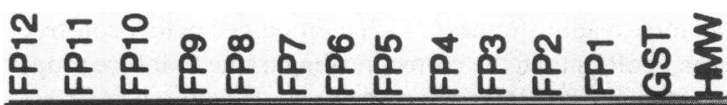

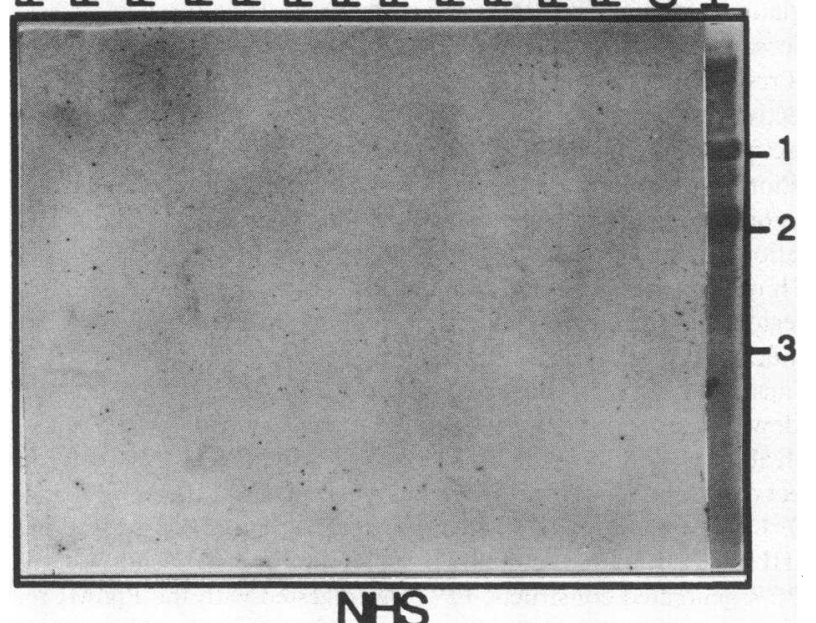

Figure 3. Western immunoblotting analyses with uncleaved recombinant fusion proteins corresponding to the NC-1, NC-2, or the collagenous domains of type VII collagen as depicted in Fig. $1 \mathrm{~A}$. The fusion protein tested is indicated on the top of each lane. HMW in each frame represents the $M_{\mathrm{r}}$ markers on the corresponding gel: $1,116 \mathrm{kD} ; 2,80 \mathrm{kD} ; 3,49.5 \mathrm{kD}$. H3A and L3D are two monoclonal antibodies against type VII 
Table II. Reactivity of the EBA Sera (1-19) in Western Analyses with Uncleaved Fusion Proteins Corresponding to Type VII Collagen Segments

\begin{tabular}{|c|c|c|c|c|c|c|c|c|c|c|c|c|c|c|c|c|c|c|c|c|c|}
\hline & \multirow{2}{*}{$\begin{array}{l}\text { Fusion } \\
\text { protein }\end{array}$} & \multicolumn{19}{|c|}{ EBA sera } & \multirow[b]{2}{*}{ Total } \\
\hline & & 1 & 2 & 3 & 4 & 5 & 6 & 7 & 8 & 9 & 10 & 11 & 12 & 13 & 14 & 15 & 16 & 17 & 18 & 19 & \\
\hline \multirow{9}{*}{ NC-1 } & FP1 & ++ & ++ & + & + & + & - & ++ & ++ & ++ & - & + & ++ & ++ & - & ++ & + & + & ++ & ++ & $16 / 19$ \\
\hline & FP2 & - & - & - & - & - & - & - & - & - & - & - & - & - & - & - & - & - & - & - & $0 / 19$ \\
\hline & FP3 & - & ++ & ++ & ++ & - & + & ++ & + & - & - & - & - & - & - & - & ++ & - & ++ & - & $8 / 19$ \\
\hline & FP4 & ++ & - & + & + & ++ & ++ & ++ & + & + & ++ & ++ & ++ & ++ & ++ & - & ++ & ++ & ++ & ++ & $17 / 19$ \\
\hline & FP5 & - & - & + & + & ++ & + & ++ & + & + & - & + & - & - & ++ & - & ++ & - & ++ & - & $11 / 19$ \\
\hline & FP6 & - & - & - & - & + & + & - & + & + & - & + & - & - & - & - & + & - & + & - & $7 / 19$ \\
\hline & FP7 & - & - & + & + & ++ & + & ++ & + & + & - & + & - & - & ++ & - & + & - & + & - & $11 / 19$ \\
\hline & FP8 & ++ & - & + & + & + & + & - & + & + & ++ & ++ & ++ & ++ & + & - & ++ & ++ & - & + & $15 / 19$ \\
\hline & FP9 & - & - & - & - & - & - & - & - & - & - & - & - & - & - & - & - & - & - & - & $0 / 19$ \\
\hline \multirow[t]{2}{*}{ Collagenous domain } & FP10 & - & - & - & - & - & - & - & - & - & - & - & - & - & - & - & - & - & - & - & $0 / 19$ \\
\hline & FP11 & - & - & - & - & - & - & - & - & - & - & - & - & - & - & - & - & - & - & - & $0 / 19$ \\
\hline $\mathrm{NC}-2$ & FP12 & - & - & - & - & - & - & - & - & - & - & - & - & - & - & - & - & - & - & - & $0 / 19$ \\
\hline
\end{tabular}

The symbols,+++ , and - indicate strongly positive, positive, and negative staining, respectively. The total number of positive sera of the total 19 tested is indicated on the right.

To characterize the protein fragments corresponding to type VII collagen sequences, the fusion proteins were cleaved by digestion with thrombin (Sigma), $30 \mathrm{U} / \mathrm{ml}$, for $2 \mathrm{~h}$ at $25^{\circ} \mathrm{C}$, giving rise to the GST and the isolated recombinant type VII collagen peptides. The efficiency of thrombin cleavage was determined by the shift in the mobility of the immunoreactive protein in Western blots or by staining the SDS-polyacrylamide gels with Coomassie Blue in case the protein was not immunoreactive (Table I).

\section{Results}

Preparation of proteins for epitope mapping. All EBA sera tested in this study contained anti-basement membrane zone antibodies that $(a)$ labeled the basement membrane zone between the epidermis and dermis by immunostaining, $(b)$ exclusively labeled the dermal side of salt-split human skin substrate, and $(c)$ reacted with the $290-300-\mathrm{kD} \alpha 1$ (VII) collagen chain by Western immunoblots of proteins extracted from human cutaneous basement membranes and proteins precipitated from conditioned medium of WISH cells $(6,13,14)$. As illustrated by Fig. 2, the nonhelical, bacterial collagenase-resistant NC-1 domain $\left(M_{\mathrm{r}} \sim 145 \mathrm{kD}\right)$ was readily recognized by all EBA sera tested (lane 2, panel $A$ ). In contrast, the type VII collagen $\alpha$-chain devoid of the NC-1 domain, (which was removed by pepsinization), was not recognized by any of the EBA sera (Fig. 2, lane 2, panel $B$ ).

Using the pGEX expression vector annealed with the original $\alpha 1$ (VII) collagen cDNA FP4 (11), its truncated versions, or PCR products derived from segments of cloned type VII collagen cDNAs, we produced a series of fusion proteins encompassing $\sim 80 \%$ of the human type VII collagen molecule (Fig. $1 A$ ). These fusion proteins include the nonhelical NC-1 and NC-2 domains, as well as most of the triple-helical collagenous domain, including the central noncollagenous 39-amino acid "hinge" region. The synthesized fusion proteins had mo- lecular masses that were within the range of their predicted sizes, as estimated by SDS-PAGE (Table I) (19). Each fusion protein also consisted of GST $\left(M_{\mathrm{r}} 26 \mathrm{kD}\right)$ and a thrombin-sensitive site (six amino acids) linked to the amino-terminal end of the type VII collagen peptide segment. The proteins were expressed from the pGEX vector which was designed in this manner to allow purification of the GST-fusion proteins by affinity chromatography on a glutathione column (21). The fact that the molecular migration of the fusion proteins purified by the column chromatography shifted downward after cleaving the GST by treatment with thrombin assured that each fusion protein was indeed the product of transcription of the recombined pGEX-type VII collagen gene segments ( Table I).

It is important to emphasize that in this prokaryotic system, post-translational modifications of the fusion proteins, such as hydroxylation, glycosylation, and enzymatic cross-linking, do not occur. Furthermore, the immunoblotting analyses were performed after denaturation of the protein in SDS. Thus, the epitopes recognized by the EBA sera consist of continuous primary sequences of the $\alpha 1$ (VII) collagen chains, independent of secondary or tertiary structures and of post-translational modifications.

Identification of antigenic regions within type VII collagen. Western immunoblots of the uncleaved fusion proteins with the 19 EBA sera tested demonstrated that there are multiple antigenic epitopes within the type VII collagen molecule that are recognized by the autoantibodies in these sera (Fig. 3 and Table II). Minor, lower molecular weight bands in the blots of some of the fusion proteins represent degradation of the full length products, as attested by a shift to lower molecular weights after digestion with thrombin.

All antigenic epitopes were confined to the nonhelical domains of the molecule, NC-1. Specifically, each one of the 19

collagen. EBA 1 and EBA 7 represent patients' serum number 1 and 7 in table II, respectively. BSLE 1 represent patient serum number 1 in Table III. A control reaction with normal human serum $(N H S)$ as well as glutathione-S-transferase $(G S T)$, an integral part of the fusion protein, demonstrates negative staining. 
A.

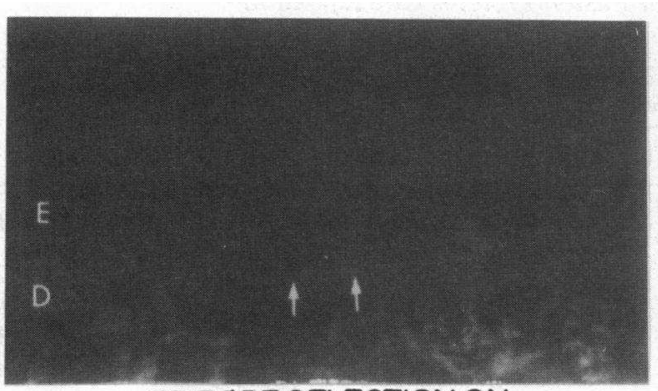

EPITOPE SELECTIONON

FP1 OF SERUM \#1

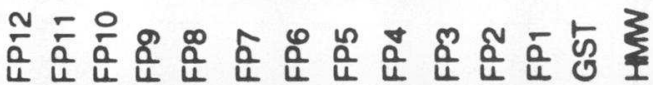

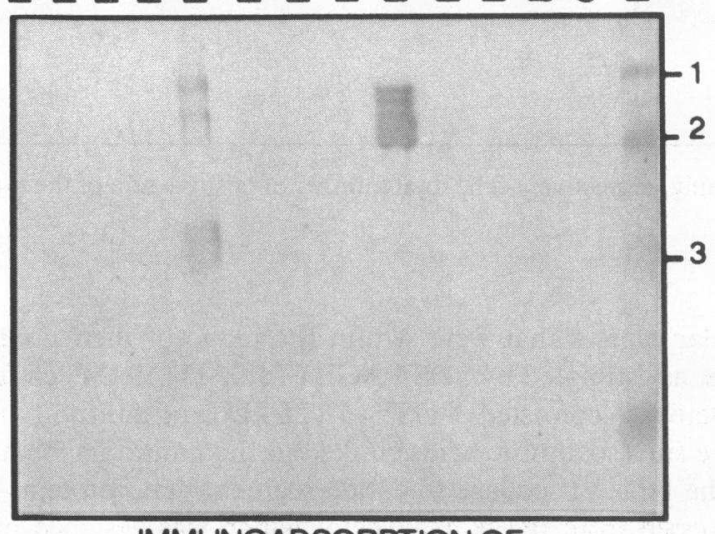

IMMUNOADSORPTION OF

SERUM \#1 ON FP1

C.

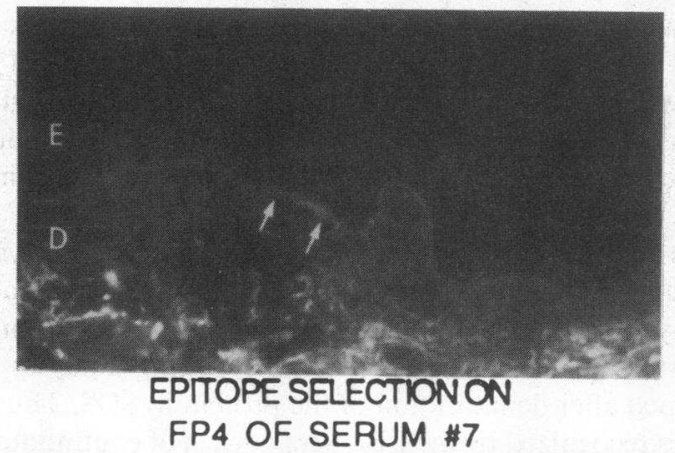

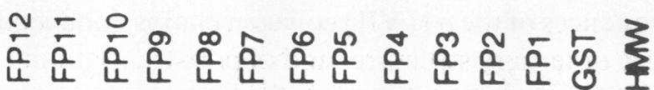

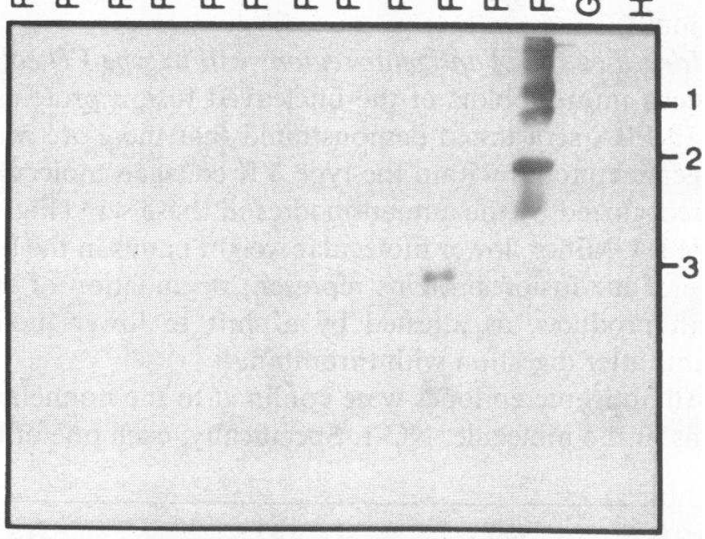

IMMUNOADSORPTION OF

SERUM \#7 ON FP4
B.

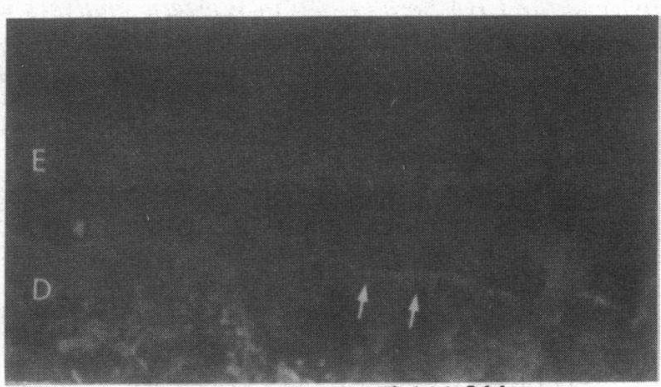

EPTOPE SELECTIONON

FP4 OF SERUM \#1

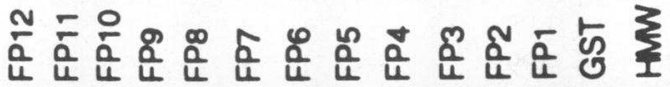

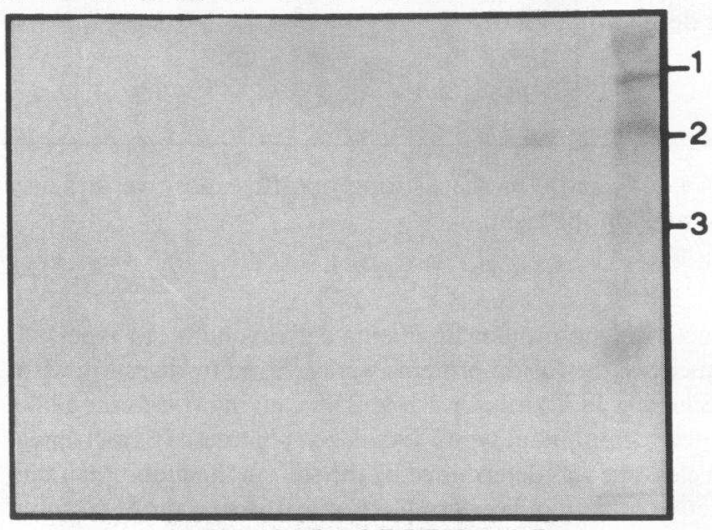

IMMUNOADSORPTION OF

SERUM 1 ON FPA

D.

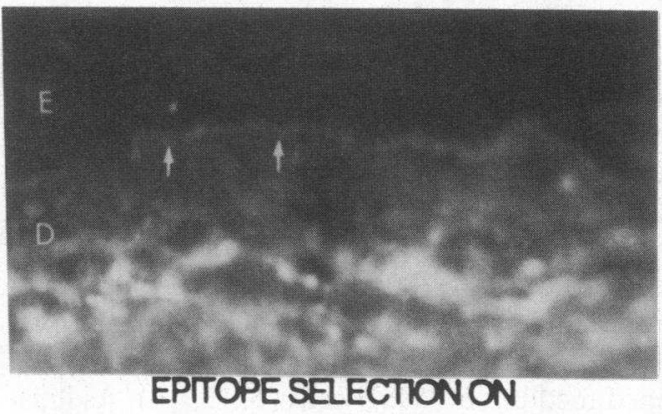

FP2 OF SERUM $\# 7$
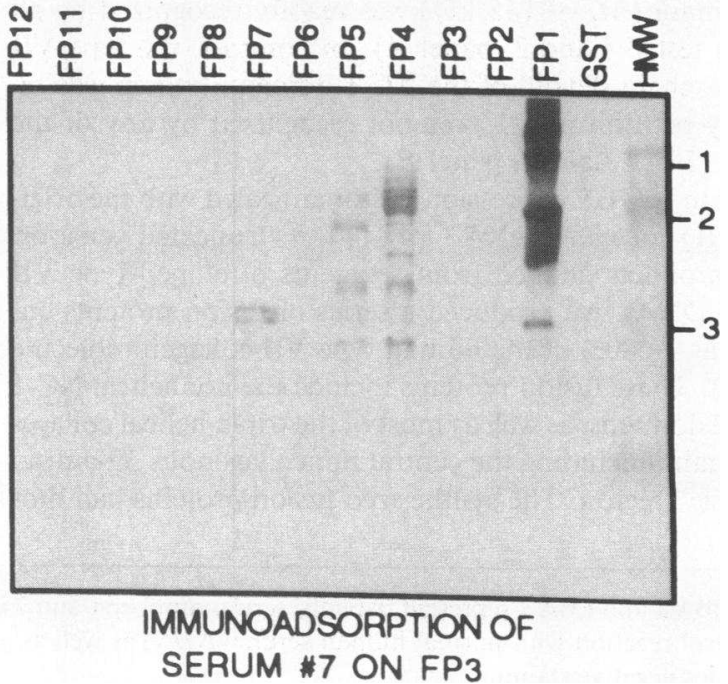
Table III. Reactivity of the BSLE Sera (1-3) in Western Analyses with Uncleaved Fusion Proteins Corresponding to Type VII Collagen Segments

\begin{tabular}{|c|c|c|c|c|c|}
\hline & \multirow{2}{*}{$\begin{array}{l}\text { Fusion } \\
\text { protein }\end{array}$} & \multicolumn{3}{|c|}{ BSLE sera } & \multirow[b]{2}{*}{ Total } \\
\hline & & 1 & 2 & 3 & \\
\hline \multirow{9}{*}{ NC-1 } & FP1 & ++ & - & ++ & $2 / 3$ \\
\hline & FP2 & - & - & - & $0 / 3$ \\
\hline & FP3 & - & - & - & $0 / 3$ \\
\hline & FP4 & ++ & + & ++ & $3 / 3$ \\
\hline & FP5 & ++ & + & - & $2 / 3$ \\
\hline & FP6 & + & - & - & $1 / 3$ \\
\hline & FP7 & ++ & + & - & $2 / 3$ \\
\hline & FP8 & ++ & + & ++ & $3 / 3$ \\
\hline & FP9 & - & - & - & $0 / 3$ \\
\hline \multirow[t]{2}{*}{ Collagenous domain } & FP10 & + & - & - & $1 / 3$ \\
\hline & FP11 & + & - & - & $1 / 3$ \\
\hline NC-2 & FP12 & + & - & - & $1 / 3$ \\
\hline
\end{tabular}

The symbols,+++ , and - indicate strongly positive, positive, and negative staining, respectively. The total number of the positive sera of the total three tested is indicated on the right.

EBA sera tested reacted with one or more different epitopes within the NC-1 domain (Table II and Fig. $1 B$ ). Within the NC-1 domain, most of the fusion proteins recognized by the EBA autoantibodies corresponded to the fibronectin type IIIlike repeat region $(8,12)$. Other subdomains outside of this area, but within the NC-1 domain, such as the region with homology to the A domain of von Willebrand factor (vWF-A), were also recognized by several sera. Three distinct immunodominant epitopes within the fibronectin type III domain, as defined by fusion proteins FP1, FP3, and the carboxyl-terminal half of FP7, were detected. These three epitopes were recognized by 18 out of 19 EBA sera tested (Table II). The absence of cross reactivity among these three epitopes was demonstrated by immunoabsorption and immunofluorescence with affinity selected antibodies (Fig. 4) and also by the unique pattern of reactivity obtained with each individual serum. For example, serum 1 reacted only with FP1 whereas serum 2 reacted with FP1 and FP3, and serum 14 reacted with FP7 (Table II). Therefore, although these antigenic domains are confined within the fibronectin type III-like repeat region of the NC-1 domain, they are apparently distinct.

Another immunodominant epitope was defined by reactivity with fusion proteins FP8 and FP4, but not with FP5 (Fig. 1). 7 of 19 EBA sera, including serum 10, which did not react with other epitopes within the NC-1 domain, showed strong labeling in this region (Table II). This epitope corresponds to the carboxyl-terminal portion of the NC-1 domain with homol- ogy to the VWF-A sequence, and the cysteine-proline-rich region between the collagenous domain and the VWF-A region (Fig. 1).

The antibodies were also tested for reactivity with three fusion proteins, FP9, FP10, and FP11, which correspond to the helical collagenous domain of the protein. No sera demonstrated reactivity with fusion proteins corresponding to the collagenous domain (Table II). Furthermore, as indicated above, Western immunoblotting of the pepsin-resistant collagenous domain with 19 sera also yielded negative labeling (Fig. $2 \mathrm{~B}$ ). Thus, the triple-helical portion of type VII collagen does not contain major antigenic epitopes that can be recognized by EBA.

Taken together, these experiments make it clear that EBA autoantibodies are polyclonal and recognize multiple distinct epitopes across the span of the $\alpha 1$ (VII) collagen chain, the major epitopes being within the noncollagenous NC-1 domain.

Western analyses with sera from patients with BSLE and other blistering skin diseases. Previous studies have shown that sera from patients with BSLE may contain autoantibodies to type VII collagen, while sera from SLE patients without blisters do not (15). Three sera from patients with BSLE were examined in addition to the EBA sera. All three BSLE sera clearly recognized type VII collagen epitopes confined to NC-1 similar to that observed with the EBA sera (Table III, Figs. 1 and 3 ). Thus, many of the same epitopes are recognized by BSLE and EBA autoantibodies.

In contrast to EBA and BSLE sera, the sera from normal subjects and patients with bullous pemphigoid or various forms of pemphigus (see Methods) did not react with any of the fusion proteins tested. The absence of reactivity of the fusion proteins with normal control sera or sera from patients with other bullous diseases attests to the specificity of the BSLE and EBA autoantibodies for type VII collagen.

Epitope mapping of two monoclonal antibodies. In addition to the autoantibodies in the EBA sera, we examined two monoclonal anti-type VII collagen antibodies, H3A and L3D (15, 18). By Western blot analysis, these two monoclonal antibodies reacted with the collagenase-resistant NC-1 domain and not with the collagenous portion of the molecule (data not shown). As expected with monoclonal antibodies, H3A and L3D demonstrated a more restricted epitope pattern within the type VII molecule than the EBA autoantibodies. Specifically, the L3D antibody did not recognize any fusion protein outside of FP4, and reacted with the fusion protein FP7, but not FP6 (Fig. 3). These observations imply that the epitope for L3D must reside within the 75-amino acid region at the carboxyl end ( 3 ' region) of the peptide segment encoded by FP7. Furthermore, the monoclonal antibody $\mathrm{H} 3 \mathrm{~A}$ recognized only the fusion protein FP1 (Fig. 3). It is of interest that these two monoclonals react within the same regions on the type VII collagen molecule as the EBA sera, confirming the highly antigenic nature of these two regions (Fig. $1 B$ ).

Figure 4. Western immunoblotting analyses with immunoadsorbed serum and indirect immunofluorescence with the epitope-selected antibodies. $(A$ and $B$ ) EBA serum number 1 of Table II and Fig. 3. ( $C$ and $D$ ) EBA serum number 7 of Table II and Fig. 3. Each lower Western blot panel demonstrates the epitopes recognized after immunoadsorption of the sera onto a nitrocellulose filter immobilized with the indicated fusion protein. Note that after immunoadsorption, sera do not label the fusion protein used for the immunoadsorption, but still label other immunoreactive fusion proteins (see the Western blot of EBA 1 and EBA 7 in Fig. 3 for the complete reactivity against all the fusion proteins). Also, when the autoantibodies bound to the fusion protein are eluted and then used for indirect immunofluorescence on $1 \mathrm{M} \mathrm{NaCl} \mathrm{salt} \mathrm{split} \mathrm{skin,} \mathrm{they} \mathrm{label} \mathrm{the}$ dermal side of the dermal-epidermal junction $(D)$ but not the epidermal side $(E)$, identical to whole EBA sera. 


\section{Discussion}

In this study, we examined discrete domains within the $\alpha 1$ (VII) chain of type VII collagen for their ability to be recognized by the autoantibodies in the sera of patients with EBA and BSLE. All EBA sera, which contained autoantibodies to the cutaneous basement membrane zone, as determined by indirect immunofluorescence staining of human skin, recognized one or more of the subdomains of type VII collagen by Western immunoblotting.

All EBA sera tested recognized the collagenase-resistant NC-1 domain $\left(M_{\mathrm{r}} \sim 145 \mathrm{kD}\right)$, while no sera reacted with the pepsinized type VII molecule corresponding to the collagenous peptide segment. These observations suggest that the nonhelical domains of type VII collagen are more antigenic than the triple-helical collagenous domain. This suggestion also is consistent with previous demonstrations that the nonhelical telopeptides of type I collagen or the propeptides of type I procollagen are more antigenic than the collagenous segment (23).

These findings using native type VII collagen were further extended by studying the reactivity of a series of fusion proteins synthesized from discrete domains of the human type VII collagen gene using a recombinant expression vector (21). These fusion proteins span $\sim 80 \%$ of the $\alpha 1$ (VII) collagen chain. The results indicated that all EBA sera recognized more than one epitope. However, the fibronectin type III-like repeat segments within the NC-1 domain were the most frequently recognized region. Although antigenic epitopes within the fibronectin type III-like repeat region of the type VII collagen were the most frequently recognized regions by the EBA sera, it is important to note that EBA autoantibodies do not recognize fibronectin itself (6). Differential labeling of the panel of fusion proteins by EBA sera (Table II), as well as independent immunoadsorption to the four different antigenic domains, clearly excludes a repeating antigenic motif within the fibronectin type III-like repeat of the NC-1 domain. This set of results clearly identifies four distinct, independent epitopes within the amino terminus NC-1 domain recognized by EBA sera. A panel of sera from normal individuals, as well as from patients with other blistering disorders were used as control and did not recognize any of the subdomains of type VII collagen.

In addition, all three BSLE sera tested recognized similar type VII collagen epitopes as the EBA sera (Table II and Table III). Consequently, the bullous lesions in SLE, in many if not all cases, may be associated with a similar immunologic pathogenesis as noted in EBA blisters.

EBA is thought to be an autoimmune disease (4). Although attempts to transfer passively the disease by injecting human antibodies into SCID mice with a human skin graft have been unsuccessful $(24,25)$, it has been suggested that the autoantibodies play a role in the pathogenesis of the disease and that they are involved in mechanisms of epidermal-dermal disadherence in these patients $(26,27)$. For example, type VII collagen has affinity for dermal fibronectin, a large noncollagenous glycoprotein. Thus, autoantibodies in EBA patients targeted against anchoring fibril collagen may interfere with the association between type VII collagen and fibronectin (27). This may, in part, explain the mechanism of blister formation in EBA patients.

In the case of heritable forms of dystrophic EB, it is clear that genetic alterations which disturb the synthesis of type VII collagen or its assembly into functional anchoring fibrils, can explain the clinical phenotype $(28,29)$. In the case of acquired EB, the basis for the development of autoantibodies is not clear, and there is no evidence for altered type VII collagen in EBA thus far. In this context, one hypothesis on the origin of an autoimmune disease relates to "molecular mimicry" $(30,31)$. In the case of EBA, the cross-reacting autoantibodies could prevent the assembly of type VII collagen into anchoring fibrils, interfere with the natural maintenance and turnover of anchoring fibrils, or interfere with the ability of the anchoring fibrils to stabilize the attachment of the basement membrane onto the underlying dermal connective tissues.

Our data argue against molecular mimicry as the basis of EBA, since we have identified EBA and BSLE autoantibodies that recognize multiple, apparently unrelated epitopes within the type VII collagen $\alpha$-chain. It is possible, however, that the initial insult to the anchoring fibrils, mediated by an antibody recognizing a single epitope, could lead to exposure of additional epitopes and subsequent development of polyvalent antisera. Alternatively, the development of autoantibodies recognizing type VII collagen epitopes in patients with EBA may result from altered immunologic recognition of "self," in a manner similar to that seen in SLE and other autoimmune diseases $(32,33)$. In this context, it is of interest that patients with BSLE, a subset of SLE who develop blisters in their skin, also develop autoantibodies recognizing type VII collagen epitopes (14). As shown in this study, these patients' sera contain anti-type VII collagen antibodies that recognize similar epitopes as EBA antibodies, and these patients develop bullous lesions similar to those seen in EBA. These observations underscore the possibility that the circulating autoantibodies to type VII collagen are pathogenic in EBA.

\section{Acknowledgments}

We thank the following individuals for their help in this study: Dr. George Giudice, Medical College of Wisconsin, provided the modified pGEX vector. Drs. John Stanley, National Institutes of Health, and Jean-Claude Bystryn, New York University, kindly contributed some EBA sera for this study. Monoclonal antibodies to human keratin were a generous gift of Dr. Tung-Tien Sun, New York University. We also thank Ms. Eileen O'Shaughnessy and Ms. Debra Pawlicki for preparing the manuscript.

This study was supported in part by U.S. Public Health Service, National Institutes of Health grants P01-AR38923, T32-AR07561, and R01-AR33625, and by the Dermatology Foundation.

\section{References}

1. Fine, J.-D., E. A. Bauer, R. A. Briggaman, D. M. Carter, R. A. J. Eady, N. B. Easterly, K. A. Holbrook, S. Hurwitz, L. Johnson, A. Lin, et al. 1991. Revised clinical and laboratory criteria for subtypes of inherited epidermolysis bullosa: a consensus report by the subcommittee on Diagnosis and Classification of the National Epidermolysis Bullosa Registry. J. Am. Acad. Dermatol. 24:119-135.

2. Uitto, J., E. A. Bauer, and A. N. Moshell. 1992. Symposium on epidermolysis bullosa: molecular biology and pathology of the cutaneous basement membrane zone. J. Invest. Derm. 38:391-395.

3. Lin, A. N., and D. M. Carter. 1992. Epidermolysis Bullosa: Basic and Clinical Aspects. Springer-Verlag, Inc., New York. 1-302.

4. Gammon, W. R., R. A. Briggaman, D. T. Woodley, P. W. Heald, and C. E. Wheeler, Jr. 1984. Epidermolysis bullosa acquisita-a pemphigoid-like disease. J. Am. Acad. Dermatol. 11:820-832.

5. Woodley, D. T., W. R. Gammon, and R. A. Briggaman. 1992. Epidermolysis bullosa acquisita. In Epidermolysis Bullosa: Basic and Clinical Aspects. A. N. Lin and D. M. Carter, editors. Springer-Verlag, Inc., New York. 76-86.

6. Woodley, D. T., R. A. Briggaman, E. J. O'Keefe, A. O. Inman, L. L. Queen, and W. R. Gammon. 1984. Identification of the skin basement membrane autoantigen in epidermolysis bullosa acquisita. N. Engl. J. Med. 310:1007-1013. 
7. Sakai, L. Y., D. R. Keene, N. P. Morris, and R. E. Burgeson. 1986. Type VII collagen is a major structural component of anchoring fibrils. J. Cell Biol. 103:1577-1586.

8. Uitto, J., L. C. Chung-Honet, and A. M. Christiano. 1992. Molecular biology and pathology of type VII collagen. Exp. Dermatol. 1:2-11.

9. Nieboer, C., D. M. Boorsma, M. J. Woerdeman, and G. L. Kalsbeck. 1980. Epidermolysis bullosa acquisita: immunofluorescence, electron microscopic and immunoelectron microscopic studies in four patients. Br. J. Dermatol. 102:383392.

10. Tidman, M. J., and R. A. J. Eady. 1985. Evaluation of anchoring fibrils and other components of the dermal-epidermal junction in dystrophic epidermolysis bullosa by quantitative ultrastructural technique. J. Invest. Dermatol. 84:373-377.

11. Parente, M. G., L. C. Chung, J. Ryynänen, D. T. Woodley, K. C. Wynn, E. A. Bauer, M.-G. Mattei, M.-L. Chu, and J. Uitto. 1991. Human type VII collagen: cDNA cloning and chromosomal mapping of the gene. Proc. Natl. Acad. Sci. USA. 88:6931-6935.

12. Christiano, A. M., L. M. Rosenbaum, L. C. Chung-Honet, M. Gabriela Parente, D. T. Woodley, T.-C. Pan, R. Z. Zhang, M-L. Chu, R. E. Burgeson, and J. Uitto. 1992. The large non-collagenous domain (NC-1) of type VII collagen is amino-terminal and chimeric: homology to cartilage matrix protein, the type III domains of fibronectin and the A domains of von Willebrand factor. Hum. Mol. Genet. 1:457-481.

13. Woodley, D. T., R. E. Burgeson, G. P. Lunstrum, L. Bruckner-Tuderman, M. J. Reese, and R. A. Briggaman. 1988. Epidermolysis bullosa acquisita antigen is the globular carboxyl terminus of type VII procollagen. J. Clin. Invest. 81:683687.

14. Yoshiike, T., D. T. Woodley, and R. A. Briggaman. 1988. Epidermolysis bullosa acquisita antigen: relationship between the collagenase-sensitive and -insensitive domains. J. Invest. Dermatol. 90:127-133.

15. Gammon, W. R., D. T. Woodley, K. C. Dole, and R. A. Briggaman. 1985. Evidence that antibasement membrane zone antibodies in bullous eruption of systemic lupus erythematosus recognize epidermolysis bullosa acquisita autoantigens. J. Invest. Dermatol. 84:472-476.

16. Anhalt, G. J., S. C. Kim, J. R. Stanley, N. J. Korman, D. A. Jabs, M. Kory, H. Izumi, H. Ratrie, D. Mutasim, and L. Ariss-Abdo. 1990. Paraneoplastic pemphigus: an autoimmune mucocutaneous disease associated with neoplasia. $N$. Engl. J. Med. 323:1729-1735.

17. Fullerton, S. H., D. T. Woodley, B. R. Smoller, and G. J. Anhalt. 1992. Paraneoplastic pemphigus with autoantibody deposition in bronchial epithelium: occurrence in a patient after autologous bone marrow transplantation. $J A M A(J$. Am. Med. Assoc.). 267:1500-1502, 1992.

18. Paller, A. S., L. L. Queen, D. T. Woodley, E. J. O'Keefe, W. R. Gammon, and R. A. Briggaman. 1985. A mouse monoclonal antibody against a newly discovered basement membrane component, the epidermolysis bullosa acquisita antigen. J. Invest. Dermatol. 84:215-217.
19. Laemmli, U. K. 1970. Cleavage of structural proteins during the assembly of the head of bacteriophage T4. Nature (Lond.). 227:680-685.

20. Towbin, H., T. Staehelin, and J. Gordon. 1979. Electrophoretic transfer of proteins from polyacrylamide gels to nitrocellulose sheets: procedures and some applications. Proc. Natl. Acad. Sci. USA. 76:4350-4354.

21. Smith, D. B., and K. S. Johnson. 1989. Single-step purification of polypeptides expressed in Escherichia coli of fusions with glutathione S-transferase. Gene. 67:31-40.

22. Sanger, F., S. Nicklen, and A. R. Coulson. 1977. DNA sequencing with chain terminating inhibitors. Proc. Natl. Acad. Sci. USA. 14:5463-5467.

23. Timpl, R. 1976. Immunological studies on collagen. In Biochemistry of Collagen. G. N. Ramachandran and A. H. Reddi, editors. Plenum Press, New York. 319-365.

24. Kim, Y. H., D. T. Woodley, K. C. Wynn, W. Giomi, and E. A. Bauer. 1992. Recessive dystrophic epidermolysis bullosa phenotype is preserved in xeongrafts using SCID mice: development of an experimental in vivo model. J. Invest. Dermatol. 98:191-197.

25. Chen, D., E. A. Welsh, D. T. Woodley, K. C. Wynn, R. A. Briggaman, and Y. H. Kim. 1992. Passive transfer of epidermolysis bullosa acquisita antibody into xenotransfused SCID mice with human grafts. J. Invest. Dermatol. 98:589. (Abstr.).

26. Gammon, W. R., A. O. Inman, and C. E. Wheeler, Jr. 1984. Differences in complement-dependent chemotactic activity generated by bullous pemphigoid and epidermolysis bullosa acquisita immune complexes: demonstration by leukocyte attachment and organ culture methods. J. Invest. Dermatol. 83:57-61.

27. Woodley, D. T., E. J. O'Keefe, J. A. McDonald, M. J. Reese, R. A Briggaman, and W. R. Gammon. 1987. Specific affinity between fibronectin and the epidermolysis bullosa acquisita antigen. J. Clin. Invest. 179:1826-1830.

28. Bruckner-Tuderman, L. 1991. Collagen of the dermo-epidermal junction: role in bullous disorders. Eur. J. Dermatol. 1:89-100.

29. Uitto, J., and A. M. Christiano. 1992. Molecular genetics of the cutaneous basement membrane zone: perspectives to epidermolysis bullosa and other blistering skin diseases. J. Clin. Invest. 90:687-692.

30. Maul, G. G., S. A. Jimenez, E. Riggs, and D. Zienmnicka-Kotula. 1989. Determination of an epitope of the diffuse systemic sclerosis marker antigen DNA topoisomerase I: sequence similarity with retroviral p30 gag protein sug gests a possible cause for autoimmunity in systemic sclerosis. Proc. Natl. Acad. Sci. USA. 86:8492-8496.

31. Guldner, H. H., H. J. Netter, C. Szostecki, H. J. Lakomek, and H. Will. 1988. Epitope mapping with a recombinant human $68-\mathrm{kDa}$ (U1) ribonucleoprotein antigen reveals heterogeneous autoantibody profiles in human autoimmune sera. J. Immunol. 141:469-475.

32. Sinha, A. A., M. T. Lopez, and H. O. McDevitt. 1990. Autoimmune diseases: the failure of self tolerance. Science (Wash. DC). 248:1380-1388.

33. Wraith, D. C., H. O. McDevitt, L. Steinman, and H. Acha-Orbea. 1989. T cell recognition as the target for immune intervention in autoimmune disease. Cell. 57:709-715. 